\title{
THE MARYLAND ELECTRON RING FOR INVESTIGATING SPACE-CHARGE DOMINATED BEAMS IN A CIRCULAR FODO SYSTEM*
}

\author{
$\underline{\text { M. Reiser }}^{\dagger}$, P.G. O’Shea, R.A. Kishek, S. Bernal, P. Chin, S. Guharay, Y. Li, M. Venturini, \\ J.G. Wang, V. Yun, W. Zhang, Y. Zou, M. Pruessner, Univ. of Maryland, College Park, MD, \\ T. Godlove, D. Kehne, P. Haldemann, FM Technologies, Inc., \\ R. York, D. Lawton, L. G. Vorobiev, Michigan State University, \\ I. Haber, NRL, Washington, DC, and H. Nishimura, LBNL, Berkeley, CA
}

\begin{abstract}
The University of Maryland Electron Ring (UMER), currently under construction, has been designed to study the physics of space-charge dominated beams at extremely large values of the betatron tune shift which exceed those of existing strong-focusing synchrotrons and rings by more than an order of magnitude. In this paper, the unique design features of this research facility, the new beam physics to be investigated, and recent experimental results in the injector prototype as well as simulation studies will be reviewed.
\end{abstract}

\section{INTRODUCTION}

The University of Maryland Electron Ring (UMER), currently under construction, has been designed to study the physics of space-charge dominated beams with extremely large tune shift far beyond the operating regime of existing synchrotrons and storage rings. The project is being developed with the collaboration of FM Technologies, Inc., Michigan State University, NRL and LBNL.

Unique design features of the facility include a strongfocusing lattice with printed-circuit quadrupoles and dipoles, compensation of the earth magnetic field with specially designed Helmholtz coils, single-turn injection and extraction with the use of pulsed Panofsky quads and dipoles. Three induction gaps provide fast-rising electric fields to prevent erosion of the bunch ends due to spacecharge forces as well as acceleration from $10 \mathrm{keV}$ to 50 $\mathrm{keV}$ over 100 turns in a future extension of the UMER operation. The operating regime can be changed from the extreme end of space-charge domination and large tune depression towards the low currents and small tune shifts of existing rings. UMER is a compact, low-cost university facility which will be dedicated entirely to physics research. It will provide a unique testbed for comparing and validating computer simulation codes and theory with experimental results.

\section{BEAM PHYSICS}

The relative importance of space charge in the behavior of charged particle beams propagating through transport sys-

\footnotetext{
* Work supported by the U. S. Department of Energy

† Email: mreiser@glue.umd.edu
}

tems can be understood from the smooth matched-beam envelope equation [1]

$$
k_{0}^{2} a-\frac{K}{a}-\frac{\epsilon^{2}}{a^{3}}=0 .
$$

Here, $a$ is the effective beam radius, $k_{0}$ the wavenumber of the betatron oscillation without space charge, $K=\left(I / I_{0}\right)\left(1 / \beta^{3} \gamma^{3}\right)$ the generalized perveance, $I_{0} \approx$ $(1 / 30)\left(m c^{2} / q\right)=17 \mathrm{kA}$ for electrons, and $\epsilon$ the effective unnormalized emittance. Equation (1) represents the balance between the focusing force (first term), the space charge (second term), and the emittance (third term). The betatron wavenumber with space charge can be defined as

$$
k=k_{0}\left(1-K / k_{0}^{2} a^{2}\right)^{1 / 2} .
$$

The ratio $k / k_{0}$ is known as the "tune depression". In circular machines it can be written in terms of the number of betatron oscillations per revolution with $(\nu)$ and without $\left(\nu_{0}\right)$ space charge, as $\nu / \nu_{0}$, using the relations $k_{0}=\nu_{0} / R$, $k=\nu / R$, where $R$ is the mean ring radius. By introducing the dimensionless intensity parameter

$$
\chi=\frac{K}{k_{0}^{2} a^{2}},
$$

one can express the tune depression in the form

$$
\frac{k}{k_{0}}=\frac{\nu}{\nu_{0}}=(1-\chi)^{1 / 2} .
$$

When space charge is a dominant effect in the beam physics, deviations from the equilibrium state and temperature anisotropies lead to rapid relaxation via plasma waves and instabilities (see Ch. 6 in Ref. 1). For these processes, the plasma frequency $\omega_{p}$, or the corresponding plasma oscillation wavenumber $k_{p}=\omega_{p} / v$ (where $v$ is the particle velocity) play an important role. The ratio $k_{p} / k_{0}$, or, in circular machines, the ratio of the number of plasma oscillations per revolution $\left(\nu_{p}\right)$ and $\nu_{0}$, i.e. $\nu_{p} / \nu_{0}$, can be expressed in terms of the intensity parameter $\chi$ as

$$
\frac{k_{p}}{k_{0}}=\frac{\nu_{p}}{\nu_{0}}=(2 \chi)^{1 / 2} .
$$

The plot of $\nu / \nu_{0}$ and $\nu_{p} / \nu_{0}$ versus $\chi$, shown in Fig. 1, provides a convenient roadmap for characterizing the relative importance of space charge and emittance (or temperature) in the beam physics. When space charge is negligible 
$(\chi=0)$, we have $\nu / \nu_{0}=1$ and $\nu_{p} / \nu_{0}=0$; hence, the beam radius $a$ is entirely determined by the emittance, and from Eq. (1) it is given by $a=\left(\epsilon / k_{0}\right)^{1 / 2}$. At the other extreme end of the parameter regime, where $\chi=1.0$, the beam is laminar, the emittance is zero $(\epsilon=0)$, the beam radius is given by $a=K^{1 / 2} / k_{0}, \nu=0$, and $\nu_{p}=\sqrt{2} \nu_{0}$. For $\chi=0.5$, the space-charge and emittance terms in the envelope equation are equal, and $\nu / \nu_{0}=\sqrt{0.5}$, while $\nu_{p} / \nu_{0}=$ 1.0 , or $\nu_{p}=\sqrt{2} \nu$. Thus, for the range $0 \leq \chi \leq 0.5$, we can say that the beam radius (hence the beam physics) is emittance-dominated, while for $0.5<\chi \leq 1.0$ the beam radius (physics) is space-charge dominated.

Since $K$ decreases rapidly with increasing energy, space-charge effects are most important at nonrelativistic energies near the particle sources and in the injector linacs for high-energy accelerators. High-energy synchrotrons and storage rings, on the other hand, operate in the emittance-dominated regime. To avoid dangerous resonance traversal, the intensity parameter is limited so that the tune shift values $\Delta \nu=\nu_{0}-\nu$ are typically between 0.25 and 0.50 . The corresponding values for the tune depression depend on $\nu_{0}$. For high energy rings (AGS, Tevatron, etc.) they are in the range $0.97<\nu / \nu_{0}<0.92$, $0.05<\chi<0.15$, which is marked in Fig. 1. The largest tune shift of $\Delta \nu=1.9$ was achieved in a rapid-bunching experiment at the AGS (where $\nu_{0}=8.84$ ) by the late A. Maschke and collaborators in 1977 [2]. The corresponding tune depression of $\nu / \nu_{0}=0.79$ at $\chi=0.376$ is also indicated in Fig. 1.

UMER is designed to explore the intensity regime far beyond Maschke's milestone, namely, in the extreme region of a highly space-charge dominated beam where no experiments exist at the present time. Initial operation is aimed at a high beam intensity where $\nu / \nu_{0}=0.25$. In future experiments, the intensity parameter $\chi$ will be decreased and the working point will be moved along the $\nu / \nu_{0}$, and $\nu_{p} / \nu_{0}$ curves towards Maschke's limit, and even further as indicated in Fig. 1. This is accomplished by decreasing the beam current from $100 \mathrm{~mA}$ to $25 \mathrm{~mA}$ or less in our specially designed variable perveance electron gun [3] and/or by increasing the beam energy via acceleration in the ring from $10 \mathrm{keV}$ to $50 \mathrm{keV}$ over about 100 turns.

The unknown territory in the extreme space-charge dominated regime will be very challenging and should provide a wealth of new phenomena. The UMER

facility will allow us to investigate emittance growth due to conversion of free energy, halo formation, and equipartitioning in a circular machine. So far these effects have only been studied in linear transport lines. In addition, UMER will permit experimental investigations of longitudinaltransverse coupling and beam profile changes due to dispersion, the behavior of bunch ends, resonance traversal, the longitudinal resistive wall instability, and other effects in the space-charge dominated regime that is currently inaccessible. The results will provide a better understanding of the role of space charge in bending systems and circular machines and should be of great value for the design of

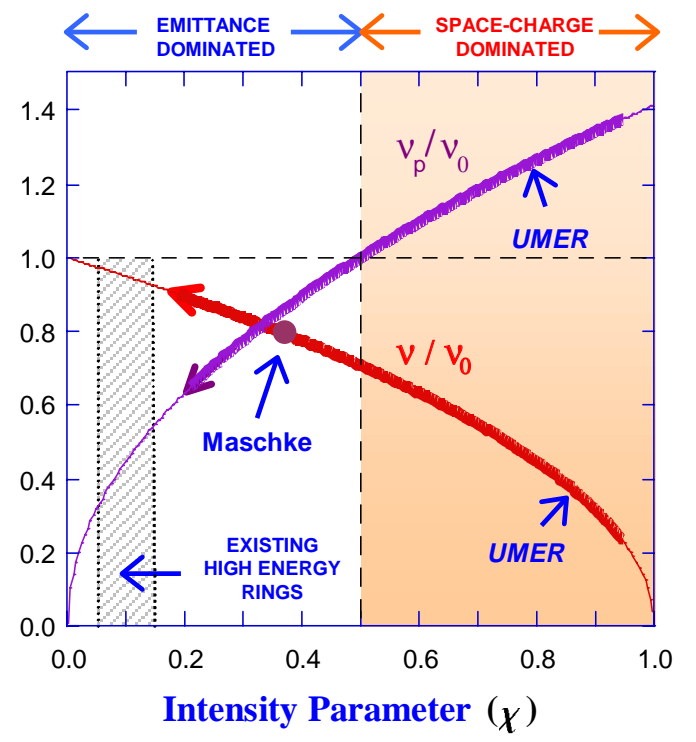

Figure 1: Beam physics regimes: Betatron and plasma oscillations vs. intensity parameter $\chi$.

future rapid cycling, high-intensity rings.

\section{DESIGN FEATURES}

A schematic layout of UMER is shown in Fig. 2. The focusing lattice consists of 36 FODO periods of length 0.32 $\mathrm{m}$ and the ring circumference is $11.52 \mathrm{~m}$. Each FODO section contains two printed quadrupole magnets and one printed dipole. The zero-current phase advance per peiod is $\sigma_{0}=76^{\circ}$, corresponding to a tune of $\nu_{0} \approx 7.6$. The maximum tune depression due to space charge is expected to be between 0.2 and 0.3 , depending on the emittance.

There are 15 diagnostic parts containing capacitive BPMs [4] and phosphor screens; three induction modules provide fast-rising "ear fields" to prevent expansion of the bunch ends [5] and acceleration in a future extension of ring operation. The electron bunch is injected into the ring at a repetition rate of $60 \mathrm{~Hz}$ or less from the injector system [3] with the help of two pulsed Panofsky quads [6] and a pulsed dipole. The bunch can be extracted within the first turn or after any number of turns with a system that duplicates the features of the injector line except that the electron gun is replaced by a large diagnostic chamber with phosphor screen, emittance meter and energy analyzer. Completion of the ring and of the first revolution of the beam is expected in late 2001 or early 2002. More details on the ring design can be found in other papers presented at this conference, and on our website: www.ipr.umd.edu/ebte/ring.

\section{PROTOTYPE EXPERIMENTS AND THEORETICAL STUDIES}

A test facility was built to investigate the performance of the printed-circuit quads and study beam transport through 


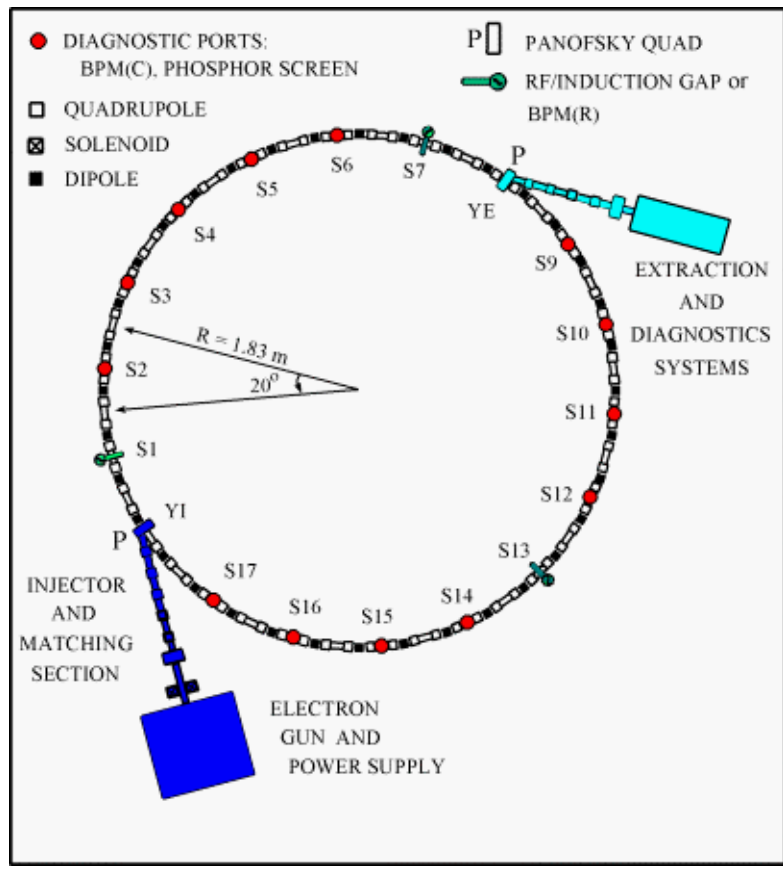

Figure 2: UMER layout.

the injector and a $20^{\circ}$ prototype sector of the ring. The experimental results were compared with simulation studies using the WARP code [8]. Agreement was excellent and gave us confidence in the extensive simulation work on beam propagation through the ring itself [9]. A new theoretical model of space-charge dominated beams in a circular lattice was used to derive a set of coupled rms envelope equations for studying rms beam behavior in rings and bending lines and for the design of combined betatron and dispersion matching for beam injection into a ring [10].

A major discovery in the prototype injector experiments was the observation of transverse space-charge waves propagating from the outer edge of the beam inward toward the center [11]. In simulation studies with the WARP code these waves were reproduced and followed over distances extending far beyond the injector length. The electron beam is passing through an aperture at the anode to reduce the beam current. The aperture cuts off the radial tails of the particle distribution, and the resulting perturbation relaxes toward equilibrium as the radial waves propagate inward and the amplitude is attenuated.

Experimental tests of the prototype $20^{\circ}$ bending section with the electron beam from the injector showed excellent performance with no beam quality deterioration or particle losses.

\section{SUMMARY}

UMER will occupy a new extreme of space-charge dominated beam physics by offering a unique opportunity to study beams of unprecedented intensity and tune depression with strong focusing and dispersion, involving physics relevant to colliders, heavy ion inertial fusion, high inten- sity rings, etc. Preparatory test experiments have already shown exciting new transverse wave phenomena. Injector experiments are planned for the summer of 1999 and experiments will begin in the fall with the first ring segments. A staged program of experiments leading to single turn operation will be completed by 2001 .

\section{REFERENCES}

[1] M. Reiser, Theory and Design of Charged Particle Beams (New York: John Wiley \& Sons, 1994).

[2] G. Danly, E. Gill, J. Keane, and A. W. Maschke, "Preliminary Results of $100 \mathrm{MeV}$ Bunching Experiment," Rep. BNL 50643, March 1, 1977.

[3] D. Kehne, et al., "The $10 \mathrm{keV}$ injector for the University of Maryland Electron Ring Project," WEA36, this conference.

[4] Y. Zou, et al., "Development of a prototype capacitive BPM," WEA96, this conference.

[5] Y. Li et al., "Calculation of particle motion at the head and tail of a bunch for the University of Maryland Electron Ring," TUP76, this conference.

[6] Y. Li et al., "Design, simulation and test of Panofsky quadrupole," THP169, this conference.

[7] W. W. Zhang, "Magnetic field measurement of printed circuit quadrupoles and dipoles," THP170, this conference.

[8] S. Bernal et al., "Transport of a space-charge dominated electron beam in a short-quadrupole channel," Phys. Rev. ST Accel. Beams, 4, 044202 (1998).

[9] R. Kishek, "PIC code simulations of collective effects in the space-charge dominated beam of the University of Maryland Electron Ring,” TUP118, this conference.

[10] M. Venturini et al., "rms envelope equations in the presence of space charge and dispersion," Phys. Rev. Lett. $\underline{81}$, 96 (1998); M. Venturini et al., "The problem of dispersion matching in space charge dominated beams," THP134, this conference.

[11] S. Bernal et al., "Observations and simulations of transverse density waves in a collimated space-charge dominated electron beam," to appear in Phys. Rev. Lett. (See also TUP115, this conference.) 